\title{
Pilot Studies of Cherry Juice Concentrate for Gout Flare Prophylaxis
}

\section{Naomi Schlesinger ${ }^{1 *}$, Ruth Rabinowitz ${ }^{2}$ and Michael Schlesinger ${ }^{3}$}

${ }^{1}$ Professor of Medicine, Chief, Division of Rheumatology, Department of Medicine, UMDNJ-Robert Wood Johnson Medical School, New Brunswick, NJ, USA ${ }^{2}$ Lecturer, Hubert H. Humphrey Center for Experimental Medicine and Cancer Research, The Hebrew University-Hadassah Medical School, Jerusalem, Israel ${ }^{3}$ Professor of Experimental Medicine and Cancer Research, Hubert H. Humphrey Center for Experimental Medicine and Cancer Research, The Hebrew University Hadassah Medical School, Jerusalem, Israel

\begin{abstract}
The management of gout involves treating pain and inflammation associated with acute flares and lowering the uric acid pool. A challenge associated with the successful management of gout is an increased risk of acute flares after initiation of urate-lowering therapy (ULT). Prophylactic anti-inflammatory therapy is recommended to prevent flares and foster compliance with urate lowering therapy.
\end{abstract}

The aim of our studies was to assess whether use of cherry juice concentrate is useful for gout flare prophylaxis.

We report the results of three studies using cherry juice concentrate for gout prophylaxis. The first is a randomized controlled study comparing the use of cherry juice concentrate versus pomegranate juice concentrate for flare prophylaxis. The second is a retrospective study evaluating flare prophylaxis when cherry juice concentrate was taken over a 4 month period or longer. Lastly, a third study evaluating the effect of cherry juice concentrate compared with pomegranate juice concentrate on secretion of interleukins by human monocytes exposed to monosodium urate (MSU) crystals in vitro.

Ingesting cherry juice concentrate reduced the incidence of flares in gout patients regardless of whether or not they were treated with ULT. The number of flares was further reduced by cherry juice ingestion in patients receiving ULT than in patients not on ULT. We did not find a significant change in serum urate levels from baseline following intake of the cherry juice concentrate for 4 months or longer. Thus, cherry juice concentrate was most likely contributing to a reduction in flares via anti-inflammatory actions. We found cherry juice concentrate to inhibit in vitro secretion of IL-1 $1 \beta$ by up to $60 \%$.

In conclusion, our studies suggest that, consumption of cherry juice concentrate for a period of 4 months or longer, reduces acute gout flares, via anti-inflammatory actions such as inhibition of IL-1 $\beta$ secretion. Large long-term randomized controlled trials are needed to further evaluate the usefulness of cherries and cherry juice concentrate for gout flare prophylaxis.

Keywords: Acute gout; Cherries; Serum urate

\section{Introduction}

Gout is the most common inflammatory arthritis in men and older women. The management of gout involves treating pain and inflammation associated with acute flares and lowering the uric acid pool. A challenge associated with the successful management of gout is an increased risk of acute flares after initiation of urate-lowering therapy (ULT). Prophylactic anti-inflammatory therapy is recommended to prevent flares and foster compliance with urate lowering therapy [1]. This can compromise patient adherence to ULT and hence adversely affect the outcome of treatment [2]. Prophylactic low-dose antiinflammatory therapy is recommended to prevent flares and foster compliance with ULT.

Prophylactic administration of low doses of colchicine and/or nonsteroidal anti-inflammatory drugs (NSAIDs) is recommended when initiating ULT in order to decrease the risk of gout flares [3]. Although, use of low doses of colchicine has been the standard of care for gout prophylaxis, there is limited data regarding the efficacy of colchicine given chronically for gout prophylaxis [4]. The recommendations for colchicine prophylaxis are based on results from placebo-controlled studies that show patients receiving colchicine prophylaxis to experience fewer flares compared with patients receiving placebo [5,6]. NSAIDs, too, are used for gout prophylaxis [7] although they have not been widely studied in clinical trials. However, safety concerns, contraindications, intolerance or lack of efficacy, limit regular use of NSAIDs or colchicine as prophylaxis in many patients with gout.
The connection of gout and hyperuricemia with gluttony, alcohol and obesity dates from ancient times [8]. Decreasing consumption of meat, seafood and alcoholic beverages as well as increasing consumption of dairy products, and tofu may be helpful in reducing gout [8]. It has been suggested in the popular press and to a lesser extent in the scientific literature that consumption of cherries alleviates arthritic pain and gout. In a 1950 paper stating that "observations made by responsible physicians suggest" that in 12 patients with gout, eating half a pound of fresh cherries or canned cherries decreased serum urate and prevented flares of gout [9]. Only three case histories are described in the paper. Not all three ate cherries persistently. In addition, there have been several anecdotal reports of the use of cherries as an effective treatment of gout; however, these reports have not been confirmed in controlled nutritional studies. The mechanism of action of cherries

*Corresponding author: Naomi Schlesinger, Professor of Medicine, Chief Division of Rheumatology, Department of Medicine, UMDNJ-Robert Wood Johnson Medical School MEB 468, One Robert Wood Johnson PI., P.O. Box 19, New Brunswick, NJ 08903-0019, USA, Tel: (732) 235-8378; Fax: (732) 235-7238; E-mail: schlesna@umdnj.edu

Received February 13, 2012; Accepted February 20, 2012; Published February 22,2012

Citation: Schlesinger N, Rabinowitz R, Schlesinger M (2012) Pilot Studies of Cherry Juice Concentrate for Gout Flare Prophylaxis. J Arthritis 1:101. doi:10.4172/2167-7921.1000101

Copyright: @ 2012 Schlesinger N, et al. This is an open-access article distributed under the terms of the Creative Commons Attribution License, which permits unrestricted use, distribution, and reproduction in any medium, provided the original author and source are credited. 
is unclear. Anthocyanins extracted from cherries have shown antiinflammatory properties and inhibited cyclooxygenase activities $[10,11]$ and modulation of tumor necrosis factor (TNF)- secretion.

The aim of our studies was to assess whether use of cherry juice concentrate is useful for gout flare prophylaxis and whether it does so via a change in serum urate (SU) or via anti-inflammatory effects.

Herein, we report the results of three studies using cherry juice concentrate for gout flare prophylaxis. The first is a randomized controlled (RCT) pilot study comparing the use of cherry juice concentrate versus pomegranate juice concentrate for gout flare prophylaxis as reflected by the number of gout flares, percent of patients with gout flares, SU level and serum creatinine when taken over a 4 month period. The second is a retrospective study evaluating flare prophylaxis when cherry juice concentrate was taken over a 4 month period or longer. Lastly, a third study evaluating the anti-inflammatory effect of cherry juice concentrate compared with pomegranate juice concentrate on the secretion of interleukins by human monocytes exposed to monosodium urate (MSU) crystals in vitro.

\section{Clinical Studies}

\section{Materials and methods (studies 1 and 2 )}

The juice concentrates used in all the studies reported here were: cherry juice concentrate prepared from fruit harvested in northern Michigan and pomegranate juice concentrate, from fruit harvested in California, both produced by Brownwood Acres Foods, Michigan. The cherry as well as the pomegranate juice concentrates contain no added sugar or sweeteners, no preservatives and no fillers. They are $100 \%$ natural juices. Each tablespoon of cherry juice concentrate is the equivalent of 45-60 cherries and each tablespoon of pomegranate concentrate equals the juice from one pomegranate. The juice concentrates were kept refrigerated until they were consumed.

Study 1: Randomized controlled study of cherry juice concentrate versus pomegranate juice concentrate for gout flare prophylaxis: Eighteen patients with MSU crystal- proven gout were entered into this study. They were randomized by blindly drawing a folded paper note assigning them to one of the two groups: group A received a tablespoon of cherry juice concentrate twice daily and group B, the control group, received a tablespoon of pomegranate juice concentrate twice daily Patients continued use of allopurinol, prophylactic colchicine or NSAIDs if they have been taking these drugs prior to initiating the study. The parameters recorded at baseline and at 120 days were the number of gout flares, percent of patients with gout flares, medications, SU level and serum creatinine.

Study 2: Retrospective study evaluating flare prophylaxis when cherry juice concentrate was taken over a 4 month period or longer: A retrospective chart review was performed of patients seen in our clinic between 7/1/04 and 5/1/09 with a primary or secondary diagnosis of either gouty arthropathy or Gout NOS, diagnosis codes 274.0 or 274.9 . The parameters recorded at baseline and after at least 4 months were the number of gout flares, percent of patients with and without gout flares, medications, SU level and serum creatinine concentration.

\section{Statistical analysis}

The Student's t-test was used to compare the different measured parameters. The significance of changes within each group was analyzed by Student's paired t-test. All $\mathrm{P}$ values were 2 -tailed and values of less than 0.05 indicate statistical significance.

\section{Results}

Study 1: Randomized Controlled trial (RCT) of Cherry juice concentrate versus pomegranate juice concentrate for gout prophylaxis: Eighteen patients with MSU crystal- proven gout were entered into this study. Four patients dropped out of the study; 2 from the cherry group ( $\mathrm{n}=1$ death prior to starting the study; $\mathrm{n}=1$ non compliant), and 2 from the pomegranate group ( $\mathrm{n}=1$ severe heartburn, $\mathrm{n}=1$ non compliant). Fourteen patients with crystal- proven gout completed an institutional review board (IRB) approved protocol. They were randomized into two groups: group $A(n=9)$ received a tablespoon of cherry juice concentrate twice daily and the control group, group $\mathrm{B}$ $(n=5)$, received a tablespoon of pomegranate juice concentrate twice daily. (Table 1)

Patient demographics included: age: range: $28-75$; $($ mean $\pm \mathrm{SE}=$ $56.43 \pm 4.10)$; disease duration: range: $3-41$; (mean $\pm \mathrm{SE}=14.43 \pm 3.04)$ : Body mass index (BMI): range: 24.4-34.4; mean $\pm \mathrm{SE}=30.02 \pm 0.84$;

\begin{tabular}{|c|c|c|c|c|}
\hline Patient number & $\begin{array}{l}\text { No. of flares before study } \\
\text { as reported by patient }\end{array}$ & $\begin{array}{l}\text { No. of flares } \\
\text { documented } \\
\text { during study }\end{array}$ & ULT/ Colchicine & NSAIDs \\
\hline \multicolumn{5}{|l|}{ Group A (Cherry) } \\
\hline 3 & 1 per month & 0 per 4 months & None & Stopped Celebrex \\
\hline 4 & 3 per month & 0 per 4 months & None & Stopped Celebrex \\
\hline 5 & 3 per month & 2 per month & Allopurinol $500 \mathrm{mg} / \mathrm{d}$, Colchicine $0.6 \mathrm{mg} / \mathrm{d}$ & No change \\
\hline 7 & 1 per month & 1 per 2 months & None & Stopped Celebrex \\
\hline 8 & 1-2 per month & 3 per 4 months & None & Stopped indomethacin \\
\hline 10 & 1-2 per month & 0 per 4 months & Allopurinol $100 \mathrm{mg} / \mathrm{d}$ & No change ${ }^{1}$ \\
\hline 11 & 1 per year & 0 per 4 months & None & No change \\
\hline 14 & 0 per 4 years & 0 per 4 months & Allopurinol 300mg /d & No change \\
\hline 16 & 2 per year & 1 per 4 months & None & Stopped indomethacin \\
\hline \multicolumn{5}{|c|}{ Group B (Pomegranate) } \\
\hline 1 & 4 per month & 3 per month & None & No change \\
\hline 2 & 1 per month & 1 per 4 months & Allopurinol $500 \mathrm{mg} / \mathrm{d}$, Colchicine $0.6 \mathrm{mg} / \mathrm{d}$ & No change \\
\hline 6 & 1-3 per year & 1 per 4 months & Allopurinol $500 \mathrm{mg} / \mathrm{d}$ & No change \\
\hline 9 & 2 per year & 0 .per 4 months. & Colchicine $0.6 \mathrm{mg} / \mathrm{d}$ & No change \\
\hline 13 & 1 per month & 1 per month & None & No change \\
\hline
\end{tabular}

1: This patient stopped taking allopurinol.

Table 1: (Study 1) Consumption of cherry juice decreases the number of gout flares and use of NSAIDs. 


\begin{tabular}{|l|c|c|c|c|c|}
\hline Patient Group & $\begin{array}{c}\text { Number of } \\
\text { patients }\end{array}$ & $\begin{array}{c}\text { Flare-free after 4 months of } \\
\text { cherry juice }\end{array}$ & $\begin{array}{c}\text { Number of flares /year prior to } \\
\text { cherry juice }\end{array}$ & $\begin{array}{c}\text { Number of flares year after } \\
\text { cherry juice }\end{array}$ & $\begin{array}{c}\text { Significance of effect of cherry } \\
\text { juice }\end{array}$ \\
\hline Not on ULT & 11 & $4 / 11(36 \%)$ & $6.82 \pm 2.10$ & $2.64 \pm 1.11$ \\
\hline On ULT & 13 & $8 / 13(62 \%)$ & $6.89 \pm 1.83$ & $1.39 \pm 0.60$ \\
\hline Total & 24 & $12 / 24(50 \%)$ & $6.85 \pm 1.34$ & $2.00 \pm \pm 0.699$ \\
\hline
\end{tabular}

Table 2: (Study 2) Consumption of cherry juice decreases the number of gout flares

;Ethnicity: Caucasian 11, Asian 1, Hispanic 1, African American 1. Three of the 9 (33\%) in group A and two of the 5 (40\%) in group B were on ULT (allopurinol 100-500 mg daily). The other patients were not on ULT due to contraindications, intolerance or lack of efficacy.

In group $\mathrm{A}$, patients consuming cherry juice concentrate the number of flares per 4 months at baseline was $4.99 \pm 1.53$ (mean \pm S. E. ) while after 120 days of cherry juice ingestion it was reduced to $1.56 \pm 0.88$ (Table 1). The difference between the number of gout flares before and after ingestion of cherry juice concentrate, assessed by 2 -tailed t-test, was significant $(\mathrm{p}<0.05)$ (Table 1$)$. In group $B$, receiving pomegranate juice concentrate the number of flares per 4 months at baseline was $5.06 \pm 2.83$ and at day 120 the mean was $3.60 \pm 2.20$. Four (45\%) patients in the group A, consuming cherry juice, versus $4(80 \%)$ patients in the group B, taking pomegranate juice, had flared during the 4 -month-study. Thus, five patients $(55 \%)$ in the cherry group were flare-free within 4 months of starting cherry juice versus only one patient (20\%) in the pomegranate group.

Five patients (55\%) in group A taking NSAIDs chronically (Celcoxib $n=3$; indomethacin $n=2$ ) discontinued NSAIDs within 60 days of starting cherry juice as opposed to none of the patients in the pomegranate group (group B).

Overall, SU levels were only slightly reduced following treatment with either cherry juice (from $8.37 \pm 0.82$ to $8.17 \pm 1.1 \mathrm{mg} / \mathrm{dL}$ ) or pomegranate juice (from $7.45 \pm 1.62$ to $6.14 \pm 1.07 \mathrm{mg} / \mathrm{dL}$ ). None of these changes were significant. In addition, there was also no significant change in serum creatinine levels in either group.

Study 2: Retrospective study evaluating flare prophylaxis when cherry juice concentrate was taken over a 4 month period or longer: We identified $24 \mathrm{MSU}$ crystal proven gout patients that were consuming cherry juice concentrate (Brownwood Acres Foods) (one tablespoon twice daily) for $\geq 4$ months. We found that the number of gout flares decreased from $6.85 \pm 1.34$ flares / year to $2.00 \pm 0.60$ flares / year (mean \pm S. E. ) ( $\mathrm{p}=0.0001$; paired two-tailed t-test $)$ in the 24 patients consuming cherry juice concentrate for $\geq 4$ months. Eleven of the 24 (45\%) gout patients were not on ULT, due to contraindications, intolerance or lack of efficacy. The mean number of gout flares a year prior to cherry juice ingestion, was $6.82 \pm 2.07$ (mean \pm S. E. ) at baseline in 11 patients not on ULT, as compared with $2.64 \pm 1.11$ after $4-6$ months of juice ingestion ( $\mathrm{p}=0.0899)$. Among 13 of the $24(55 \%)$ gout patients who were on ULT the incidence of flares was $6.89 \pm 1.83$ flares in the year prior to starting cherry juice concentrate consumption, while after consuming cherry juice concentrate for $\geq 4$ months the flare rate decreased significantly to $2.64 \pm 1.11$ / year ( $\mathrm{p}=0.0086$; paired two-tailed t-test) Table 2 .

At baseline average $\mathrm{SU}$ level in patients receiving allopurinol treatment was $8.4 \pm 0.6 \mathrm{mg} / \mathrm{dl}$, while $4-6$ months later the SU level was significantly decreased with ULT to $6.2 \pm 0.4 \mathrm{mg} / \mathrm{dl}$ ( $\mathrm{p}=0.0052)$. The average SU level at baseline in patients not on ULT was $9.0 \pm 1.1 \mathrm{mg} / \mathrm{dl}$ and remained unaltered after receiving cherry juice for 4-6 months (8.7 $\pm 1.4 \mathrm{mg} / \mathrm{dl}, \mathrm{p}=0.5943$ ). Thus, decrease of gout flares elicited by cherry juice ingestion in patients who were not on ULT was not secondary to a reduction of in the SU level.

Twelve (50\%) of the 24 patients consuming cherry juice concentrate chronically were flare-free at 4-6 months. Four of eleven (36\%) patients not taking ULT were flare-free at 4-6 months; while 8/13 (62\% ) of patients taking ULT were flare-free at 4-6 months. However the difference in the proportion of flare-free individuals between patients taking ULT and those not taking ULT was not statistically significant. The average SU among all patients who were flare-free was $7.8 \mathrm{mg} / \mathrm{dl}$

\section{Laboratory Study}

\section{Study 3: The effect of cherry juice concentrate on the secretion of interleukins by human monocytes exposed to monosodium urate crystals in vitro}

The aim of this study was to determine whether exposure of human monocytes in vitro to cherry juice concentrate affects their capacity to secrete interleukin when exposed to MSU crystals.

\section{Materials and methods (study 3 )}

Before assessing the capacity of juice concentrates to inhibit the secretion of interleukins by human monocytes we determined the cytotoxic effect of the juice concentrates on the cells employing the MTT assay. In this test, the staining of the cells by 3-(4, 5-Dimethylthiazol2-yl)-2, 5-diphenyltetrazolium bromide was determined using a microplate reader. Only dilution of juice concentrates that did not exhibit cytotoxic activity on monocytic cells were tested for their capacity to inhibit the secretion of interleukins.

The cells tested in vitro were either monocytes derived from the buffy coat of volunteer blood donors obtained from the blood bank or THP-1 cells of a monocyte-like cell line. The THP-1 cell line is a monocyte-like cell line derived from a patient with acute monocytic leukemia. Cherry juice concentrate and pomegranate juice concentrate manufactured by Brownwood Acres were tested for their capacity to inhibit interleukin secretion. MSU monohydrate crystals used for monocyte stimulation were prepared according to the method described by Schiltz et al. [12]

THP- 1 cells ( $3 \mathrm{X} 10^{6}$ cells suspended in $3 \mathrm{ml}$ culture medium) or adherent monocytes from buffy coat were introduced into $35 \mathrm{~mm}$ Petri culture dishes. After 2 hours of incubation at 37 o $\mathrm{C}$ in a $5 \% \mathrm{CO}_{2}$ humidified atmosphere the supernatant fluid was removed and $1.5 \mathrm{ml}$ MSU crystal suspension containing 100 to $250 \mu \mathrm{g} / \mathrm{ml}$ was added. Either culture medium or various concentrations of cherry or pomegranate juice were added to the Petri dishes. The cells in the culture dishes were exposed for 24 hours simultaneously to MSU crystals and to dilutions of juice concentrates at $37 \mathrm{o} \mathrm{C}$ in a $5 \% \mathrm{CO}_{2}$ humidified atmosphere. The supernatant fluid of the cultures was collected and the in vitro effect of juice concentrates on the secretion of cytokines by monocytes exposed to MSU crystals was determined. The level of both IL- $1 \beta$ and TNF- $\alpha$ in the supernatant fluids was assessed using Enzyme-linked immunosorbent assays (ELISA).

The Immunoassays were carried out with Human IL-1 $\beta / \mathrm{IL}-1 \mathrm{~F} 2$ 
and Human TNF- $\alpha$ /TNFSF1A Quantikine kits obtained from R\&D Systems, Abington OX143NB, UK. In short, $50 \mu \mathrm{L}$ assay diluent was added to each well, followed by $200 \mu \mathrm{L}$ standard, control or cell culture supernatant samples to each well. After 2 hours of incubation at room temperature the supernatant fluid in each well was aspirated, followed by 4 washes. $200 \mu \mathrm{L}$ of anti-cytokine conjugate was added to each well, incubated for 1 hour at room temperature. Thereafter the wells were washed 4 times, and $200 \mu \mathrm{L}$ substrate solution was added to each well incubated for 20 minutes, and $50 \mu \mathrm{L}$ stop solution was added to each well. The optical density of each well was determined using a microplate reader set to $450 \mathrm{~nm}$.

\section{Results}

As assessed by the MTT method, pomegranate juice concentrates were more toxic than cherry juice concentrates for human monocytes. The exposure in vitro to MSU crystals elicited secretion of IL-1 $\beta$ and TNF- $\alpha$ by either peripheral human blood monocytes or by cells of the THP-1 monocyte-like line. At a concentration that had no cytotoxic effect on monocytes (dilutions of 1:1600 or higher) cherry juice concentrate inhibited the secretion of IL-1 $\beta$ by up to $60 \%$ (Table 3a). Non-toxic concentrations of pomegranate juice had either a weak inhibitory effect or a stimulatory effect on IL-1 $\beta$ secretion. Exposure of human monocytes to cherry juice concentrate elicited an inhibitory effect on TNF- $\alpha$ secretion, while pomegranate juice had a relatively weak or no effect on TNF- $\alpha$ secretion (Table 3b).

\section{Discussion}

Gout flare prophylaxis is an integral part of chronic gout treatment. Safety concerns and contraindications limit chronic NSAIDs or colchicine use as prophylaxis in many patients with gout. Consumption of cherries and cherry products has been reported to be health promoting, particularly in alleviating arthritic pain and gout [9]. We have found cherry juice concentrate to be well tolerated and efficacious for gout flare prophylaxis.

In our prospective pilot RCT, gout patients consuming cherry juice concentrate had a significant decrease in the number of gout flares within 4 months of initiating ingestion of the cherry juice concentrate, an effect not seen in the control group, treated with pomegranate juice. Fifty five $\%$ of patients ingesting cherry juice concentrate were flare- free and stopped their NSAIDs intake. In our retrospective study of patients consuming cherry juice concentrate daily for $\geq 4$ months; a $50 \%$ or greater reduction in gout flares was seen in approximately half of the patients (similar percentage to that seen in our RCT). Importantly, 36\% of patients not taking ULT were flare-free at 4-6 months of ingesting cherry juice despite an average SU of $8.7 \mathrm{mg} / \mathrm{dl}$. Thus, although SU level in patients not taking ULT remained higher than $6.8 \mathrm{mg} / \mathrm{dL}$, the point of saturation of uric acid, cherry juice consumption significantly reduced their gout flares. Thus, ingesting cherry juice concentrate reduced the incidence of flares in gout patients regardless of whether or not they were treated with ULT.

The intake of cherries by healthy individuals was reported to provoke a significant decrease in SU levels over $5 \mathrm{~h}$ post dose, whereas other fruits such as strawberries, grapes and kiwifruit, produced no change [13]. Neither, in our pilot RCT nor in our retrospective study did we did find a significant change in SU levels from baseline following cherry juice concentrate consumption for 4 months or longer. It is therefore not a change in SU levels that contributed to the reduced gout flares in patients receiving cherry juice concentrate. However, gout patients receiving ULT were much more responsive to ingestion of cherry juice than those not receiving ULT. In our retrospective study, $36 \%$ of the gout patients who were not on ULT versus $62 \%$ who were on ULT were flare-free at 4-6 months. Thus, the number of flares was further reduced by cherry juice ingestion in patients receiving ULT than in patients not on ULT. These results indicate that, cherry juice concentrate was most likely contributed to a reduction in flares via its anti-inflammatory actions.

Both sweet and tart cherries are rich in antioxidants, including anthocyanins (responsible for red skin and flesh color), catechins, chlorogenic acid, flavonal glycosides and melatonin. Anthocyanins extracted from cherries have shown anti-inflammatory properties, via inhibition of cyclooxygenase (COX) activities [10,11]. Cherries contain natural COX 1 and COX 2 inhibitors. The COX inhibitory activities of anthocyanins from cherries were comparable to those of ibuprofen and naproxen at $10 \Phi \mathrm{M}$ concentrations. In addition, anthocyanins extracted from cherries have shown anti-inflammatory properties, via scavenging of the reactive nitric oxide (NO) radical [14]. Anthocyanins 1 and 2 are present in both cherries and raspberries. Anthocyanins and other phenolics also inhibit NO production in activated macrophages

\begin{tabular}{|c|c|c|c|c|c|c|}
\hline \multicolumn{7}{|c|}{ Effect of cherry and pomegranate juice on human monocytes stimulated by exposure to $250 \mu \mathrm{g} / \mathrm{ml} \mathrm{MSU}$} \\
\hline & Control, without juice & $\begin{array}{l}\text { Cherry juice } \\
1: 1600\end{array}$ & $\begin{array}{l}\text { Cherry juice } \\
1: 4000\end{array}$ & & $\begin{array}{l}\text { Pomegran- } \\
\text { ate juice } \\
1: 1600\end{array}$ & $\begin{array}{l}\text { Pomegran- } \\
\text { ate juice } \\
1: 4000\end{array}$ \\
\hline IL-1 ßconcentration (pg/ml) & 83.356 & 30.696 & 30.205 & & 157.768 & 229.073 \\
\hline Inhibition of IL-1 $\beta$ secretion & & $63.3 \%$ & $63.8 \%$ & & $-89.3 \%$ & $-174.8 \%$ \\
\hline IL-1 $\beta$ concentration (pg/ml) & 15.876 & 8.971 & 9.927 & & \multicolumn{2}{|l|}{21.102} \\
\hline Inhibition of IL-1 $\beta$ secretion & & $43.49 \%$ & $17.47 \%$ & & \multicolumn{2}{|l|}{$-12.92 \%$} \\
\hline
\end{tabular}

Table 3a: (Study 3) The capacity of cherry juice concentrate to inhibit the secretion of IL-1 $\beta$ by human monocytes exposed in vitro to MSU crystals.

\begin{tabular}{|l|l|l|l|l|}
\hline & Control, without juice & Cherry juice 1:4000 & Cherry juice 1:8000 & Pomegranate juice 1:8000 \\
\hline TNF- $\alpha$ concentration $(\mathrm{pg} / \mathrm{ml})$ & 52.673 & 28.579 & 33.521 & 42.170 \\
\hline Inhibition of TNF- $\alpha$ secretion & & $45.74 \%$ & $36.36 \%$ & $19.94 \%$ \\
\hline
\end{tabular}

Table 3b: (Study 3) The capacity of cherry juice concentrate to inhibit the secretion of TNF- $\alpha$ by human monocytes exposed in vitro to 250 g / ml MSU crystals. 
[14] and modulate tumor necrosis factor (TNF- $\alpha$ ) secretion [15]. Various flavons and flavonols were found to either inhibit or induce TNF- $\alpha$ production [15].

It has recently become apparent that gouty inflammation is primarily interleukin $1 \beta$ (IL-1 $\beta$ ) driven [16]. MSU crystals stimulate IL-1 release by monocytes and synovial mononuclear cells [17] as well as the cryopyrin (NLRP3) inflammasome an intracellular, multiprotein complex. Cryopyrin regulates activation of the protease caspase- 1 which in turn controls the activation of IL-1 $\beta$ [16]. The release of IL1 $\beta$ $[16,17]$ promotes neutrophil influx into the joint and joint inflammation ensues. The central role of IL-1 in the pathogenesis of gout was further demonstrated by studies of IL-1 inhibitors, showing reduction in the risk of gout flares [18].

As reported herein, we found cherry juice concentrate to inhibit the in vitro secretion of IL- $1 \beta$ by up to $60 \%$. In contrast. , non-toxic concentrations of pomegranate juice either had a weak inhibitory effect, or a slight stimulatory effect on IL-1 $\beta$ secretion. Exposure of human monocytes to cherry juice concentrate elicited a weak inhibitory effect on TNF- a secretion, while pomegranate juice had either a weak inhibitory effect or no effect.

There are a number of limitations to our studies. First, in our pilot RCT cherry juice was only given for 16 weeks. A study of a more prolonged period of cherry juice prophylaxis is needed. Secondly, our clinical studies were small, but the combination of two clinical studies reported herein, make for a stronger support of the use of cherry juice concentrate for prophylaxis flare gout.

In conclusion, our studies suggest that, consumption of cherry juice concentrate for a period of 4 months or longer, reduces acute gout flares, via anti-inflammatory actions such as inhibition of IL-1 $\beta$ secretion. Large long-term randomized controlled trials are needed to further evaluate the usefulness of cherries and cherry juice concentrate for gout flare prophylaxis.

\section{Acknowledgment}

The prospective RCT was supported by a grant from Brownwood Acres Foods. We are grateful to Paloma Levy and to Orli Marco for their skilled technical assistance.

\section{References}

1. Schlesinger $N$ (2008) Overview of the management of acute gout and the role of adrenocorticotropic hormone. Drugs 68: 407-415.

2. Schlesinger N, Dalbeth N, Perez-Ruiz F (2009) Gout--what are the treatment options? Expert Opin Pharmacother 10: 1319-1328.

3. Schlesinger N (2004) Management of acute and chronic gouty arthritis: present state-of-the-art. Drugs 64: 2399-2416.

4. Yu TF, Gutman AB (1961) Efficacy of colchicine prophylaxis in gout. Prevention of recurrent gouty arthritis over a mean period of five years in 208 gouty subjects. Ann Intern Med 55: 179-192.

5. Paulus HE, Schlosstein LH, Godfrey RG, Klinenberg JR, Bluestone R (1974) Prophylactic colchicine therapy of intercritical gout. A placebo-controlled study of probenecid-treated patients. Arthritis Rheum 17: 609-614.

6. Borstad GC, Bryant LR, Abel MP, Scroggie DA, Harris MD, et al. (2004) Colchicine for prophylaxis of acute flares when initiating allopurinol for chronic gouty arthritis. J Rheumatol 31: 2429-2432.

7. Becker MA, Schumacher HR Jr, Wortmann RL, MacDonald PA, Eustace D, et al. (2005) Febuxostat compared with allopurinol in patients with hyperuricemia and gout. N Engl J Med 353: 2450-2461.

8. Schlesinger N (2005) Dietary factors and hyperuricaemia. Curr Pharm Des 11 4133-4138.
9. Blau LW (1950) Cherry diet control for gout and arthritis. Tex Rep Biol Med 8 : 309-311

10. Wang H, Nair MG, Strasburg GM, Chang YC, Booren AM, et al. (1999) Antioxidant and antiinflammatory activities of anthocyanins and their aglycon, cyanidin, from tart cherries. J Nat Prod 62: 294-296.

11. Seeram NP, Momin RA, Nair MG, Bourquin LD (2001) Cyclooxygenase inhibitory and antioxidant cyanidin glycosides in cherries and berries. Phytomedicine 8: 362-369.

12. Schiltz C, Lioté F, Prudhommeaux F, Meunier A, Champy R, et al. (2002) Monosodium urate monohydrate crystal-induced inflammation in vivo: quantitative histomorphometric analysis of cellular events. Arthritis Rheum 46 1643-1650.

13. Jacob RA, Spinozzi GM, Simon VA, Kelley DS, Prior RL, et al. (2003) Consumption of cherries lowers plasma urate in healthy women. J Nutr 133 : 1826-1829.

14. van Acker SA, Tromp MN, Haenen GR, van der Vijgh WJ, Bast A (1995) Flavonoids as scavengers of nitric oxide radical. Biochem Biophys Res Commun 214: 755-759.

15. Wang J, Mazza G (2002) Effects of anthocyanins and other phenolic compounds on the production of tumor necrosis factor alpha in LPS/IFN-gamma-activated RAW 264.7 macrophages. J Agric Food Chem 50: 4183-4189.

16. Di Giovine FS, Malawista SE, Nuki G, Duff GW (1987) Interleukin 1 (IL 1) as a mediator of crystal arthritis. Stimulation of $T$ cell and synovial fibroblast mitogenesis by urate crystal-induced IL 1. J Immunol 138: 3213-3218.

17. Martinon F, Pétrilli V, Mayor A, Tardivel A, Tschopp J (2006) Gout-associated uric acid crystals activate the NALP3 inflammasome. Nature 440: 237-241.

18. Schumacher HR Jr, Sundy JS, Terkeltaub R, Knapp HR, Mellis SJ, et al. (2012) Rilonacept (IL-1 Trap) in the prevention of acute gout flares during initiation of urate-lowering therapy: results of a Phase 2 clinical trial. Arthritis Rheum

19. Schlesinger N, Mysler E, Lin HY, De Meulemeester M, Rovensky J, et al. (2011) Canakinumab reduces the risk of acute gouty arthritis flares during initiation of allopurinol treatment: results of a double-blind, randomised study. Ann Rheum Dis 70: 1264-1271. 